Trab-623-00 (6 páginas)

\title{
ESTRUTURA GENÉTICA ESPACIAL DE DUAS POPULAÇÕES NATURAIS DE Myracrodruon urundeuva M. Allemão NA REGIÃo SEMI-ÁRIDA, BRASIL ${ }^{1}$
}

\author{
Cristina Maria Batista de Lacerda² e Paulo Yoshio Kageyama ${ }^{3}$
}

\begin{abstract}
RESUMO - Para determinar se a distribuição espacial dos genótipos é aleatória ou se está estruturada, utilizou-se a autocorrelação espacial dos genótipos para investigar dados alozímicos de duas populações naturais de Myracrodruon urundeuva do semi-árido brasileiro (Estação Ecológica do Seridó/RN, com 1.166,38/ha e Sítio Mata dos Alves/PB com 84/ha). A primeira população encontra-se em áreas mais preservadas, enquanto a segunda está em uma área fragmentada. O programa utilizado foi o "Autocorr". A autocorrelação estimada para os locos polimórficos foi realizada nos indivíduos adultos. Foram analisados dois alelos na Estação Ecológica do Seridó e quatro no Sítio Mata dos Alves. Utilizaram-se de três métodos para o pareamento de indivíduos a serem comparados: conexão de Gabriel $\left(\mathrm{I}_{\mathrm{g}}\right)$, vizinho mais próximo $\left(\mathrm{I}_{\mathrm{vmp}}\right)$ e comparações dentro de classes de distâncias preestabelecidas. Os resultados obtidos para $\mathrm{I}_{\mathrm{g}}$ e $\mathrm{I}_{\mathrm{vmp}}$ (Pgm-2: 0,032 e 0,236; Est-1: 0,263 e 0,242, respectivamente) na estação ecológica e $I_{g}$ e $I_{\text {vmp }}$ (Pgm-1: -0,349 e -0,288; Pgm-2: -0,341 e-0,278; Est-1: -0,349 e -0,284 e Mdh-1: -0,345 e $-0,282$, respectivamente) no sítio não mostraram a presença de estruturação genética espacial, o que possibilita pressupor que exista uma distribuição aleatória dos genótipos dentro delas. O mesmo foi detectado para as comparações dentro de classes de distâncias preestabelecidas. Os resultados mantiveram o mesmo padrão encontrado para algumas populações naturais de espécies arbóreas tropicais já estudadas.
\end{abstract}

Palavras-chave: $\quad$ Myracrodruon urundeuva, populações naturais, semi-árido, genótipos, alelos e autocorrelação.

\section{SPACIAL GENOTYPE STRUCTURE OF TWO NATURAL POPULATIONS OF Myracrodruon urundeuva M. Allemão IN A SEMI-ARID REGION OF BRAZIL}

\begin{abstract}
To determine whether genotype spacial distribution is random or structured, spacial genotypic autocorrelation was used to compare two natural populations of Myracrodruon urundeuva in the semi-arid region of Northeastern Brazil (Estação Ecológica do Seridó/RN - 1166,38/ha and Sítio Mata dos Alves/PB - 84/ ha). The former is characteristic of preserved areas, while the latter is found in areas with marked anthropogenic degradation. The program "Autocorr" was used for the analysis. The autocorrelation was run on polymorphic loci detected in the adult individuals. Two alleles were analyzed at the Estação Ecológica do Seridó and 4 at the Sitio Mata dos Alves. The three methods used for analysis were: Connection of Gabriel (Ig), Closest neighbor (Ivmp) and Pre-established distance comparisons. The results obtained for Ig and Ivmp (Pgm-2: 0,032 and 0,236; Est-1: 0,263 and 0,242, respectively) in the ecological station sample/populations and Ig and Ivmp (Pgm1: -0,349 and -0,288; Pgm-2: -0,341 and 0,278; Est-1: -0,349 and -0,284 and Mdh-1: -0,345 and -0,282, respectively) in the farm sample/populations. The results did not indicate the presence of special genetic structure, making it possible to hypothesize that there is an internal randomized genotypic distribution of the genotype. The same was detected for the pre established distance comparisons. The results maintained the same pattern found in some natural populations of tropical tree species studied.
\end{abstract}

Key words: $\quad$ Myracrodruon urundeuva, natural populations, semi-arid, genotypes, alleles, and autocorrelation.

1 Recebido para publicação em 18.5.2000.

Aceito para publicação em 19.2.2003.

Parte da Dissertação de Mestrado, realizado na Escola Superior de Agricultura “Luiz de Queiroz"/USP, Piracicaba-SP.

2 Engenheira Florestal, M.S. - Escola Superior de Agricultura “Luiz de Queiroz"/USP, Piracicaba-Brasil, <crisblacerda@uol.com.br>; ${ }^{3}$ Departamento de Ciências Florestais/LARGEA/ESALQ/USP, - Av. Pádua Dias,11, Caixa Postal 09, 13418-900 Piracicaba-SP. 


\section{INTRODUÇÃO}

A variedade de vida no planeta Terra, incluindo a variabilidade genética dentro das espécies e populações de espécies (flora, fauna e de microrganismos), e as mudanças nas funções ecológicas desempenhadas pelos organismos nos ecossistemas, nas comunidades e nos habitats são denominadas de Biodiversidade ou Diversidade Biológica, por Souza Dias (1996). Esta diversidade é uma das propriedades fundamentais da natureza, responsável pelo equilíbrio e pela estabilidade dos ecossistemas, sendo o desempenho de suas funções ecológicas ainda pouco compreendido.

Essa diversidade biológica está se deteriorando (principalmente com o aumento da taxa de extinção de espécies) devido ao impacto das atividades humanas; ação esta que desequilibra e desestabiliza os ecossistemas. Portanto, para conservação genética de uma espécie é necessário o conhecimento da distribuição da variabilidade genética entre e dentro de suas populações.

Considerando que a maior parte da variabilidade genética das espécies arbóreas alógamas encontra-se dentro de populações, o conhecimento de suas causas torna-se de fundamental importância. Essa afirmação vem sendo comprovada através de estudos realizados com populações de espécies arbóreas a partir de marcadores alozímicos, que têm revelado alta variabilidade genética, principalmente dentro das populações.

Em estudos genéticos, a autocorrelação espacial pode ser utilizada tanto para estimar o grau de isolamento de populações a partir das freqüências gênicas ou fenotípicas em cada população, como em nível intrapopulacional, em que esta técnica é utilizada para auxiliar na detecção de agrupamentos de indivíduos aparentados (Gandara, 1996).

O presente estudo visou determinar se a distribuição espacial dos genótipos é aleatória ou se está estruturada em familiares, utilizando a autocorrelação espacial dos genótipos em duas populações naturais de Myracrodruon urundeuva (aroeira) do semi-árido brasileiro.

\section{MATERIAL E MÉTODOS}

\subsection{Populações}

As duas populações de aroeira estudadas são originárias da região semi-árida brasileira. A população da
Estação Ecológica do Seridó - município de Serra Negra do Norte-RN, com 1.166,38/ha (latitude $6^{\circ} 66^{\prime} 00^{\prime \prime} \mathrm{S}$ e longitude $37^{\circ} 40^{\prime} 00^{\prime \prime} \mathrm{W}$ ), encontra-se em áreas mais preservadas (maior diversidade de espécies). Já a população do Sítio Mata dos Alves - Imaculada-PB, com 84/ha (7³9'00”S e longitude 3750’00”W), localiza-se em área cujo estádio de degradação pela ação antrópica está bem acentuado, com a aroeira apresentando-se em "stands" quase puros. Com o auxílio de uma trena e uma bússola, foram mapeados 30 indivíduos adultos (reprodutivos) para o estudo da estrutura espacial da aroeira.

\subsection{Dados alozímicos}

Foram extraídas as enzimas de tecidos foliares de 30 indivíduos adultos por população, através dos seguintes sistemas isoenzimáticos: fosfoglucomutase (PGM EC 2.7.5.1); fosfoglucose isomerase (PGI - EC 5.3.1.9); 6-phosphoglucomato desidrogenase (6PGDH - EC 1.1.1.44); malato desidrogenase (MDH - EC 1.1.1.37); glutamato-oxaloacetato transaminase (GOT - EC 2.6.1.1); leucina aminopeptidase (LAP - EC 3.4.4.11.1) e $\alpha$-esterase ( $\alpha$-Est - EC 3.1.1.1) (Lacerda, 1997; Lacerda et al., 2001).

\subsection{Análise Estatística}

Para analisar a estrutura genética espacial em duas populações de $M$. urundeuva procedeu-se à análise espacial dos genótipos através da autocorrelação, com base em Sokal \& Oden (1978a, b). O programa utilizado foi o "Autocorr", confeccionado por Jonh S. Heywood.

A análise de autocorrelação considera cada alelo $\left(\mathrm{p}_{\mathrm{i}}\right)$ como uma variável, portanto cada genótipo homozigótico recebe o valor de $\mathrm{p}_{\mathrm{i}}=1,0$, o heterozigótico $\mathrm{p}_{\mathrm{i}}=$ 0,5 e quando o alelo está ausente, $\mathrm{p}_{\mathrm{i}}=$ zero. $\mathrm{O}$ número de alelos analisados por loco corresponde a $\mathrm{n}-1$, em que $n$ é o número de alelos existente. Assim, de um loco com dois alelos, apenas um deles foi avaliado, uma vez que o outro tem freqüência dependente deste. A autocorrelação foi analisada para os locos polimórficos detectados nos indivíduos adultos.

Dessa forma, foram analisados dois alelos na população da Estação Ecológica do Seridó: Pgm-2 (alelo 1) e Est-1 (alelo 1) e quatro alelos no Sítio Mata dos Alves: Pgm-1 (alelo 1), Pgm-2 (alelo 1), Est-1 (alelo 1) e Mdh-1 (alelo 1). 
A caracterização da estrutura espacial dos genótipos foi feita a partir do índice I de Moran, de acordo com Sokal \& Oden (1978a). Este índice quantificou a similaridade genética de pares de indivíduos adjacentes em relação à amostra da população como um todo. O índice I de Moran foi calculado pela fórmula a seguir, para um dado alelo:

$$
I(k)=n \sum_{i} \sum_{j(i \neq j)} w_{i j} z_{i} z_{j} /\left(S \sum_{i} z_{i}^{2}\right)
$$

em que $z_{i}=\hat{p}_{i}-\hat{p}$, sendo $\hat{p}$ a média de $\quad ; w_{i j}=1$ se a distância entre a $i$-ésima e a $j$-ésima planta for classificada dentro da classe $k$; do contrário, $w_{i j}$ é zero e n é o número total de amostras. O índice I de Moran, segundo Gandara (1996), pode assumir valores entre -1 (autocorrelação negativa, ou seja, os indivíduos pareados são completamente diferentes) e +1 (autocorrelação positiva, ou seja, os indivíduos pareados são idênticos). Valor zero significa ausência de autocorrelação, isto é, que os indivíduos estão aleatoriamente distribuídos no espaço.

Foram utilizados três métodos para o pareamento de indivíduos a serem comparados pela análise de distribuição espacial dos genótipos de M. urundeuva: conexão de Gabriel, vizinho mais próximo e comparações dentro de classes de distâncias preestabelecidas. Foram preestabelecidas 9 e 13 classes de distâncias na população da Estação Ecológica do Seridó-RN e no Sítio Mata dos Alves-PB, respectivamente, objetivando um número de conexões mínimas em torno de 30. Para maior evidência do comportamento da distribuição espacial dos genótipos por classes de distância, foram construídos correlogramas para comparar o índice I de Moran obtido para diversas classes de distância em relação às distâncias especificadas, indicando a extensão da estrutura espacial quando presente.

\section{RESULTADOS E DISCUSSÃO}

O Quadro 1 mostra os resultados relativos aos valores do índice I de Moran para a conexão de Gabriel e o vizinho mais próximo, para as duas populações naturais de M. urundeuva (Estação Ecológica do Seridó$\mathrm{RN}$ e Sítio Mata dos Alves-PB, respectivamente).

Os resultados obtidos para o índice I de Moran, utilizando os métodos de conexão de Gabriel e vizinho mais próximo, analisado na população da Estação Ecológica do Seridó (dois alelos), apesar de positivos não foram significativos, ou seja, não mostraram uma estruturação genética espacial, de onde pressupõe-se que exista uma distribuição aleatória dos genótipos. Contudo, na população do Sítio Mata dos Alves é possível observar uma mudança substancial pelos valores distanciados de zero, tendendo a uma formação de estrutura familiar. A ausência de estruturação genética espacial detectada nas populações não significa que não existam indivíduos aparentados. Alguns pesquisadores suspeitam da existência de estruturação dentro das populações naturais de plantas, contudo, para algumas populações naturais de espécies arbóreas e arbustos tropicais já estudadas, tal padrão não tem sido encontrado, como em Cedrela fissilis (Gandara, 1996), Aspidosperma polyneuron (Maltez, 1997), Genipa americana (Sebbenn, 1997), Chorisia speciosa (Souza, 1997), Psychotria nervosa (Dewey \& Heywood, 1988), Platypodium elegans (Hamrick et al., 1993) e para a média dos alelos de Cryptocarya moschata (Moraes, 1997).

Quadro 1 - Estimativa do índice I de Moran para duas populações naturais de Myracrodruon. urundeuva, através dos coeficientes de autocorrelação espacial (I de Moran) para a conexão de Gabriel ( $\left.\mathrm{I}_{\mathrm{g}}\right)$ e vizinho mais próximo ( $\mathrm{I}_{\mathrm{vmp}}$ )

Table 1 - Estimate of the Index I of Moran for two natural populations of Myracrodruon urundeuva, using coefficients of spacial autocorrelation (I Moran) for Connection of Gabriel (Ig) and Closest Neighbor (Ivmp)

\begin{tabular}{|l|c|c|c|c|c|}
\hline \multirow{2}{*}{ Loco } & \multirow{2}{*}{ Alelo } & \multicolumn{2}{|c|}{ Estação Ecológica do Seridó/RN } & \multicolumn{2}{c|}{ Sítio Mata dos Alves/PB } \\
\cline { 3 - 6 } & & $\mathrm{I}_{\mathrm{g}}$ & $\mathrm{I}_{\mathrm{vmp}}$ & $\mathrm{I}_{\mathrm{g}}$ & $\mathrm{I}_{\mathrm{vm}}$ \\
\hline Pgm-1 & 1 & & & $-0,349^{\mathrm{ns}}$ & $-0,288^{\mathrm{ns}}$ \\
Pgm-2 & 1 & $0,032^{\mathrm{ns}}$ & $0,236^{\mathrm{ns}}$ & $-0,341^{\mathrm{ns}}$ & $-0,278^{\mathrm{ns}}$ \\
Est-1 & 1 & $0,263^{\mathrm{ns}}$ & $0,242^{\mathrm{ns}}$ & $-0,349^{\mathrm{ns}}$ & $-0,284^{\mathrm{ns}}$ \\
Mdh-1 & 1 & & & $-0,345^{\mathrm{ns}}$ & $-0,282^{\mathrm{ns}}$ \\
\hline Distância média (m) & & $17,81 \pm 10,74$ & $11,17 \pm 6,03$ & $8,84 \pm 6,6$ & $5,69 \pm 4,1$ \\
\hline Número de comparações & & 46 & 21 & 30 & \multicolumn{2}{c|}{21} \\
\hline
\end{tabular}


Na população Sítio Mata dos Alves os valores foram negativos, mas não-significativos, portanto não diferentes de zero, o que indica distribuição aleatória dos genótipos.

Os índices I de Moran para as nove classes de distância na Estação Ecológica e para as 13 classes no Sítio Mata dos Alves podem ser observados nos Quadros 2 e 3 , respectivamente. A flutuação mais próxima ao valor zero reforça a idéia da distribuição espacial aleatória dos genótipos dos índices para os locos analisados, bem como para o índice médio (Figuras 1 e 2).

Dos 18 valores obtidos na Estação Ecológica do Seridó (Quadro 2) apenas quatro apresentaram-se significativos $(22,2 \%)$, o que evidencia que os indivíduos próximos tendem a ser mais aparentados nessas classes de distância (H e I).

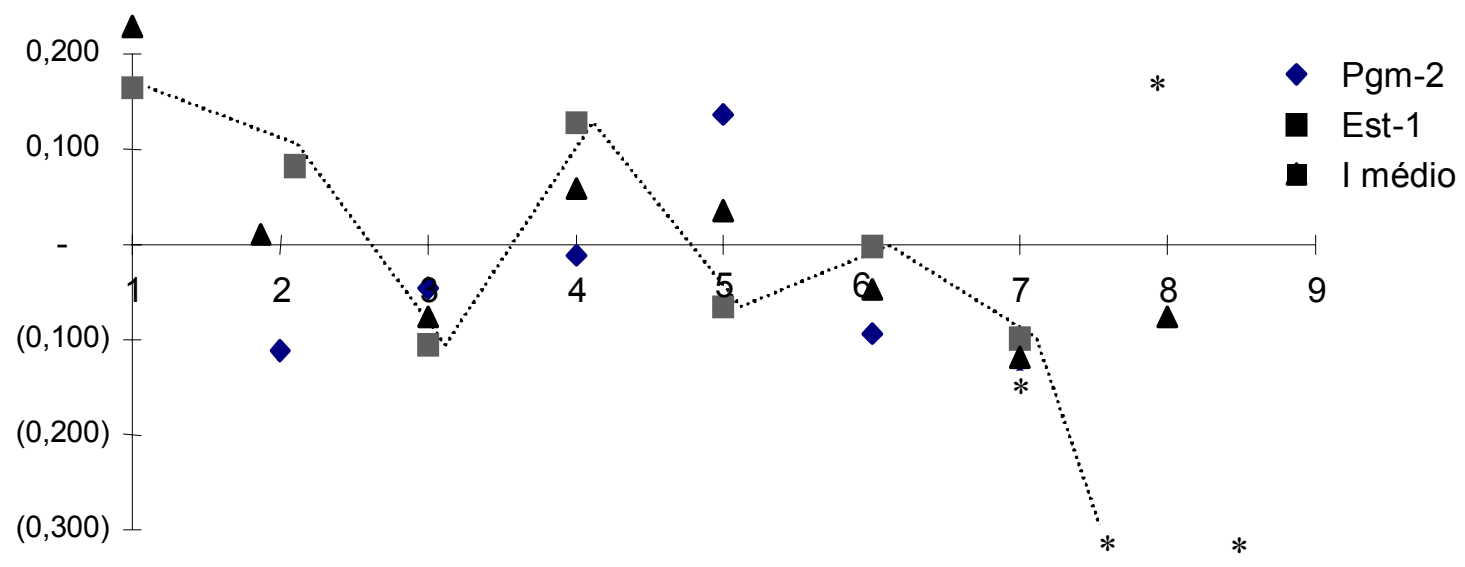

Figura 1 - Correlogramas dos alelos Pgm-2 (alelo 1) e Est-1 (alelo 1) e para a média dos dois locos, considerando nove classes de distância (* indica autocorrelação significativa), para uma população de Myracrodruon urundeuva na Estação Ecológica do Seridó/RN.

Figure 1 - Correlating the alleles Pgm-2 (allele 1) and Est-1 (allele 1) and for the average of the 2 loci considering 9 distance classes (* indicates significant autocorrelation), for a population of Myracrodruon urundeuva in the Estação Ecológica do Seridó/RN.

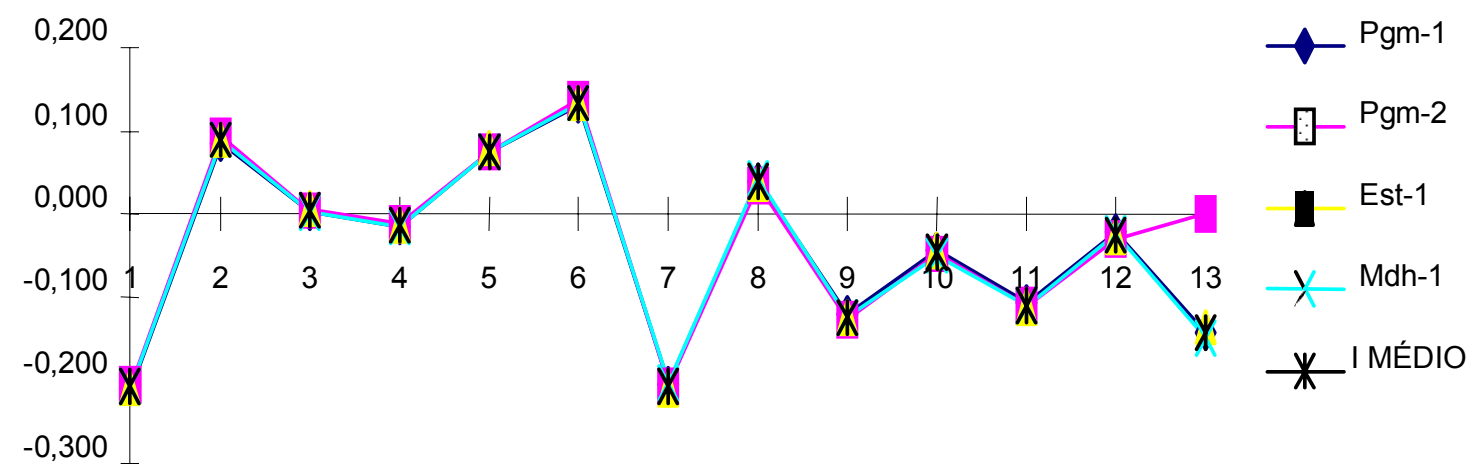

Figura 2 - Correlogramas dos alelos Pgm-1 (alelo 1), Pgm-2 (alelo 1), Est-1 (alelo 1) e Mdh-1 (alelo 1) e para a média dos quatro locos, considerando 13 classes de distância, para uma população de Myracrodruon urundeuva no Sítio Mata dos Alves-PB.

Figure 2 - Correlating the alleles Pgm-1 (allele 1), Pgm-2 (allele 1), Est-1 (allele 1) and Mdh-1 (allele 1) and for the average of the 4 loci considering 13 distance classes, for population of Myracrodruon urundeuva in the Sitio Mata dos Alves / PB.

R. Árvore, Viçosa-MG, v.27, n.2, p.145-150, 2003 
Quadro 2 - Coeficientes de autocorrelação espacial (I de Moran) para 9 classes de distância, distância média entre indivíduos pareados e número de comparações, para a população de Myracrodruon urundeuva na Estação Ecológica do Seridó/RN

Table 2 - Coefficients of spacial autocorrelation (I of Moran) for 9 distance classes, medium distance between individual pairs and number of comparisons, for the population of Myracrodruon urundeuva in the Estação Ecológica do Seridó/RN

\begin{tabular}{|l|c|c|c|c|c|c|c|c|c|c|}
\hline \multirow{2}{*}{ Loco } & \multirow{2}{*}{ Alelo } & \multicolumn{9}{|c|}{ Classe de Distância* } \\
\cline { 3 - 12 } & & A & B & C & D & E & F & G & H & I \\
\hline Pgm-2 & 1 & $0,293^{\text {ns }}$ & $-0,111^{\text {ns }}$ & $-0,046^{\text {ns }}$ & $-0,011^{\text {ns }}$ & $0,136^{\text {ns }}$ & $-0,094^{\text {ns }}$ & $-0,141^{\text {ns }}$ & $0,408^{* *}$ & $-0,966^{* *}$ \\
Est-1 & 1 & $0,164^{\text {ns }}$ & $0,104^{\text {ns }}$ & $-0,106^{\text {ns }}$ & $0,128^{\text {ns }}$ & $-0,066^{\text {ns }}$ & $-0,002^{\text {ns }}$ & $-0,099^{\text {ns }}$ & $-0,560^{* *}$ & $-0,674^{* *}$ \\
\hline I médio & & 0,229 & $-0,004$ & $-0,076$ & 0,059 & 0,035 & $-0,048$ & $-0,12$ & $-0,076$ & $-0,82$ \\
\hline \multicolumn{2}{|l}{ Distância Média (m) (DP) } & 8,71 & 23,41 & 37,35 & 53,39 & 66,28 & 82,49 & 97,19 & 111,40 & 126,03 \\
& $(3,55)$ & $(3,98)$ & $(4,39)$ & $(4,17)$ & $(4,33)$ & $(4,47)$ & $(4,35)$ & $(4,03)$ & $(4,76)$ \\
\hline
\end{tabular}

ns (não-significativo); **P $<0,01 ; \mathrm{DP}=$ desvio-padrão.

* Classe de distância em metros: A, 0-15; B, 15-30; C, 30-45;D, 45-60; E, 60-75; F, 75-90; G, 90-105; H, 105-120; I, 120-135.

Quadro 3 - Coeficientes de autocorrelação espacial (I de Moran) para 13 classes de distância, distância média entre indivíduos pareados e número de comparações, para uma população de Myracrodruon urundeuva no Sítio Mata dos Alves/PB

Table 3 - Coefficients of spacial autocorrelation (I of Moran) for 13 distance classes, medium distance between individual pairs and number of comparisons, for the population of Myracrodruon urundeuva in the Sitio Mata dos Alves/PB

\begin{tabular}{|c|c|c|c|c|c|c|c|c|c|c|c|c|c|c|}
\hline \multirow{2}{*}{ Loco } & \multirow{2}{*}{ Alelo } & \multicolumn{13}{|c|}{ Classe de Distância* } \\
\hline & & $\mathrm{A}$ & $\mathrm{B}$ & $\mathrm{C}$ & $\mathrm{D}$ & $E$ & $F$ & G & $\mathrm{H}$ & $\bar{I}$ & $\overline{\mathrm{J}}$ & $\bar{L}$ & $\mathrm{M}$ & $\mathrm{N}$ \\
\hline Pgm- $1^{\frac{1}{}}$ & 1 & $-0,210$ & 0,086 & 0,003 & $-0,016$ & 0,075 & 0,132 & $-0,206$ & 0,039 & $-0,121$ & $-0,044$ & $-0,107$ & $-0,021$ & $-0,143$ \\
\hline Pgm- $2^{\frac{1}{}}$ & 1 & $-0,205$ & 0,093 & 0,004 & $-0,011$ & 0,074 & 0,137 & $-0,207$ & 0,035 & $-0,126$ & $-0,048$ & $-0,111$ & $-0,030$ & -0148 \\
\hline Est-1 ${ }^{1 /}$ & 1 & $-0,209$ & 0,090 & 0,006 & $-0,014$ & 0,079 & 0,134 & $-0,211$ & 0,036 & $-0,125$ & $-0,043$ & $-0,113$ & $-0,027$ & $-0,137$ \\
\hline Mdh- $1^{\frac{1}{}}$ & 1 & $-0,208$ & 0,089 & 0,003 & $-0,016$ & 0,075 & 0,134 & $-0,203$ & 0,043 & $-0,125$ & $-0,049$ & $-0,111$ & $-0,024$ & $-0,148$ \\
\hline I médio & & $-0,208$ & 0,089 & 0,004 & $-0,014$ & 0,076 & 0,134 & $-0,207$ & 0,038 & $-0,124$ & $-0,046$ & \begin{tabular}{|l|}
$-0,111$ \\
\end{tabular} & $-0,026$ & $-0,144$ \\
\hline $\begin{array}{l}\text { Distância Média } \\
\text { (m) (DP) }\end{array}$ & & $\begin{array}{l}08,09 \\
(4,27) \\
\end{array}$ & $\begin{array}{l}24,65 \\
(4,29) \\
\end{array}$ & $\begin{array}{r}30,47 \\
(4,7) \\
\end{array}$ & $\begin{array}{l}55,60 \\
(4,74) \\
\end{array}$ & $\begin{array}{l}72,20 \\
(4,72) \\
\end{array}$ & $\begin{array}{l}87,85 \\
(4,58) \\
\end{array}$ & $\begin{array}{l}102,77 \\
(4,44) \\
\end{array}$ & $\begin{array}{l}119,85 \\
(4,70) \\
\end{array}$ & \begin{tabular}{|l|}
135,99 \\
$(4,94)$ \\
\end{tabular} & $\begin{array}{l}151,15 \\
(4,49) \\
\end{array}$ & \begin{tabular}{|l|}
180,63 \\
$(11,07)$ \\
\end{tabular} & $\begin{array}{r}215,31 \\
(8,91) \\
\end{array}$ & \begin{tabular}{|l|}
254,70 \\
$(11,93)$ \\
\end{tabular} \\
\hline $\begin{array}{l}\text { Número de } \\
\text { comparações }\end{array}$ & & 40 & 55 & 42 & 50 & 50 & 29 & 34 & 36 & 29 & 30 & 54 & 31 & 16 \\
\hline
\end{tabular}

${ }^{1 /}$ Todos os locos apresentaram valores de I não-significativos; DP = desvio-padrão.

* Classes de distância em metros: A,0-16; B, 16-32; C, 32-48; D, 48-64; E, 64-80; F, 80-96; G, 96-112; H, 112-28; I, 128-144; J, 144$160 ; \mathrm{L}, 160-200 ; \mathrm{M}, 200-240 ; \mathrm{N}, 240-280$.

Ao analisar as médias dos valores do índice I de Moran na população da Estação Ecológica, nos dois locos em cada classe de distância (Figura 1), observa-se que o índice mantém-se praticamente constante, demonstrando assim a não-formação de uma estrutura espacial significativa, a não ser para as duas últimas classes de distância, não desconsiderando, portanto, que existem alguns indivíduos próximos, relacionados geneticamente, responsáveis por alguns cruzamentos endogâmicos, expressos pelas diferenças entre a taxa de cruzamento multilocos e unilocos (Lacerda, 1997). As médias dos quatro locos da população do Sítio Mata dos Alves, apesar das variações demonstradas, com tendência à formação de estrutura familiar (Figura 2), foram nãosignificativas, ou seja, os genótipos encontram-se distribuídos aleatoriamente dentro das populações.

A não-estruturação espacial para os alelos analisados indica que a seleção local e, ou, a deriva genética local são fracas o suficiente para não ocasionar uma estrutura significativa em presença de fluxo gênico.

R. Árvore, Viçosa-MG, v.27, n.2, p.145-150, 2003 


\section{CONCLUSÃO}

Dentro da população de adultos da Estação Ecológica do Seridó-RN e do Sítio Mata dos Alves-PB, o padrão de distribuição encontrado através da análise de autocorrelação espacial para os dados analisados mostrou aleatoriedade dos genótipos.

A população secundária (Sítio Mata dos Alves) já apresenta mudança substancial na sua estrutura, tendendo à formação de estrutura familiar.

A estrutura genética espacial é importante tanto para a conservação/manejo como para fins de melhoramento, possibilitando a formação de amostras mais significativas em populações naturais de plantas, colaborando, assim, na sustentabilidade dos recursos genéticos.

\section{REFERÊNCIAS BIBLIOGRÁFICAS}

DEWEY, S. E.; HEYWOOD, J. S. Spatial genetic structure in a population of Psychotria nervosa. I. Distribution of genotypes. Evolution, v. 42, p. 834-38, 1988.

GANDARA, F. B. Diversidade genética, taxa de cruzamento e estrutura espacial dos genótipos em uma população de Cedrela fissilis Vell. (Meliaceae). 1996. 69 f. Dissertação (Mestrado) - Universidade Estadual de Campinas, Campinas, 1996.

HAMRICK; J. L.; MURASWSKI, D. A.; NASON, J. D. The influence of seed dispersal mechanisms on the genetic structure of tropical tree populations. Vegetation, v.107/ 108, p. 281-297, 1991/1993.

LACERDA, C. M. B. Diversidade genética por isoenzimas em populações naturais de aroeira (Myracrodruon urundeuva M. Allemão) Anacardiaceae no semi-árido. 1997. 96 f. Dissertação (Mestrado) - Escola Superior de Agricultura "Luiz de Queiroz", Piracicaba, 1997.

R. Árvore, Viçosa-MG, v.27, n.2, p.145-150, 2003
LACERDA, C. M. B. et al. Definição de protocolo enzimático para estudo da estrutura genética da aroeira. Revista Árvore, v. 25, n. 3, p. 311-315, 2001.

MALTEZ, H. M. Estrutura genética de Aspidoperma polyneuron Muell. Arg. - Apocynaceae (Peroba Rosa) em uma floresta estacional semidecidua no Estado de São

Paulo. 1997. 132 f. Dissertação (Mestrado) - Universidade Estadual de Campinas, Campinas,1997.

MORAES, P. L. R. Estrutura genética de populações de Cryptocarya moschata Nees \& Martius ex Nees (Lauraceae). 1997. 197 f. Tese (Doutorado) Universidade Estadual de Paulista, Rio Claro, 1997.

SEBBENN, A. M. Estrutura genética de subpopulações de Genipa americana L. (Rubiaceae) a partir de isoenzimas. 1997. 107 f. Dissertação (Mestrado) - Escola Superior de Agricultura "Luiz de Queiroz", Piracicaba, 1997.

SOKAL, R. R.; ODEN, N. L. Spatial autocorrelation in biology I Methodology. Biolology Journal Linnux of Society, v. 10, p. 119-228, 1978a.

SOKAL, R. R.; ODEN, N. L. Spatial autocorrelation in biology 2 Some biological implications and four applications of evolutionary and ecological interest. Biology Journal Linnux Society, v. 10, p. 229-249, 1978 b.

SOUZA DIAS, B. F. Conservação da diversidade biológica. In: PUIGNAU, J. P.; CUNHA, R. (Eds.). Conservacion de germoplasma vegetal. Montevideo, PROCISUR, 1996. p. 1-6.

SOUZA, L. M. F. I. Estrutura genética de populações naturais de Chorisia speciosa A.St.-Hil. (Bombacaceae) em fragmentos florestais na região de Bauru (SP) - Brasil. 1997. 76 f. Dissertação (Mestrado) - Escola Superior de Agricultura "Luiz de Queiroz", Piracicaba, 1997. 